\title{
3D acoustic scattering from an irregular fluid waveguide via the BEM
}

\author{
L. Godinho, A. Tadeu* , F. Branco \\ Department of Civil Engineering, Faculty of Science and Technology, University of Coimbra Polo II, Pinhal de Marrocos, 3030-290 Coimbra, Portugal
}

Received 20 March 2000; revised 7 August 2000; accepted 12 February 2001

\begin{abstract}
The BEM is used to calculate the variation in the pressure field generated by a dilatational point load inside a channel filled with a homogeneous fluid, in the presence of an irregular floor. The Green's functions are defined in the frequency domain and obtained by superposing virtual acoustic sources combined so as to generate the boundary conditions of the free or rigid surfaces of the channel. The responses in the time domain are obtained by means of Fourier transforms, making use of complex frequencies. The main features and spectral representation of the signals scattered by irregular floors are then described and used to elucidate the most important aspect of wave acoustics, which can provide the basis for the development of non-destructive testing and imaging methods. (C) 2001 Elsevier Science Ltd. All rights reserved.
\end{abstract}

Keywords: Wave propagation; Seismic prospecting; Acoustic mediums

\section{Introduction}

It is important to conduct surveys of the earth's crust before proceeding with the construction of large buildings, motorways, dams and ports. Although some geological features, such as the terrain profile and the depth of the rock matrix, are routinely determined it is still not possible to detect with accuracy the presence, depth, size and mass of buried structures (cavities, foundations, tunnels or elastic inclusions).

Seismic methods are probably the most important for studying geological discontinuities [1-5]. These methods involve the generation of seismic waves (the incident field), using dynamic sources, and the measurement of spatial and temporal variations in the resulting field by means of receivers or geophones. The field scattering arises from the interaction of the incident field and inclusions, but it is strongly influenced by the arrangement of the different strata of the earth's crust. The intervals, amplitudes and phase distortions of the waves recorded on the receivers can be used to draw inferences on the geological structure of the medium.

This study analyses the alteration in the pressure field generated by a point pressure source inside a hydraulic waveguide in the presence of an irregular floor. This

\footnotetext{
* Corresponding author. Tel.: +351-239-797-201; fax: +351-239-797190.

E-mail address: tadeu@dec.uc.pt (A. Tadeu).
}

problem requires the solution of the Helmholtz equation. Many methods for solving this equation are discussed in the acoustics literature. They range from the analytical methods presented by Pao and Mow [6] for studying wave diffraction near cylindrical inclusions, to purely numerical methods, such as finite difference [7] and finite elements techniques [8], combined with transmitting boundaries. The latter have mostly been restricted to situations where the response is required only in localized irregular domains, such as for soil structure interaction problems [9].

Boundary integral equation methods form another class of techniques used to analyse acoustic scattering from surfaces or compact inclusions submerged inside a fluid medium. A half space medium [10] is usually assumed, with the pressure field satisfying a free or rigid boundary condition along the surface. Dawson [11] formulated a boundary integral equation method to compute the scattering of underwater sound from the compact deformations of an oceanic waveguide's surface. In his numerical examples, the fluid filling the waveguide is assumed to have constant density and sound speed. The solution involves a Green's function appropriate to the waveguide in the absence of the boundary deformation, allowing the sound speed to vary with depth (not illustrated in the examples). This Green's function is obtained either by taking the Fourier transform of the standard 3D model expansion [12] with respect to a transverse coordinate, or by assuming a vertical eigenfunction expansion for the Green's function and evaluating the coefficients in a manner analogous to the 3D model [12]. 
The same method was used $[13,14]$ to obtain the 2D acoustic scattered field generated by objects embedded between two half spaces with different densities.

Possibly the best way to analyse wave propagation problems in unbounded media is the boundary element method (BEM), because it automatically satisfies the far field radiation conditions and allows a compact description of the medium in terms of boundary elements at the material discontinuities alone. Although the BEM leads to a fully populated system of equations, as opposed to the sparse system given by the finite difference and finite element techniques, the technique is made efficient because it reduces computational effort. As is well known, the BEM is based on the use of appropriate fundamental solutions, or Green's functions, relating the field variables in a homogeneous medium to point sources placed somewhere within it. The fundamental solution most often used is that of an infinite homogeneous space, because it is known in closed-form and has a relatively simple structure. However, BEMs based on the Green's functions for a half-space have been used to solve problems involving wave diffraction by surface irregularities of arbitrary shape (e.g. Refs. [15-17]), as well as for cavities and buried structures (e.g. Ref. [18]).

This paper applies the BEM to the problem of the scattering of pressure waves generated by a point load inside a fluid waveguide with an irregular floor and whose geometry does not change in one direction (z). Such a situation is frequently referred to as a two-and-a-half-dimensional problem (or 2-1/2-D for short), for which solutions can be obtained by means of a spatial Fourier transform in the direction in which the geometry does not vary. This requires solving a sequence of $2 \mathrm{D}$ problems with different spatial wave numbers $k_{z}[19]$. Then, using the inverse Fourier transform, the 3D field can be synthesized.

The solution at each frequency is expressed in terms of waves with varying wave number $k_{z}$ (with $z$ being the direction in which the geometry does not vary), which is subsequently Fourier transformed into the spatial domain. The wave number transform in discrete form is obtained by considering an infinite number of virtual point sources equally spaced along the $z$-axis and at a sufficient distance from each other to avoid spatial contamination [20]. In addition, the analyses are performed using complex frequencies, shifting down the frequency axis, in the complex plane and minimizing the influence of the neighbouring fictitious sources [21].

This attenuation greatly helps to reduce the number of spatial virtual sources needed to build the Green's function. Indeed, given the slight shift of the frequency axis downwards, that is, by considering complex frequencies, the spatial virtual sources that occur at times later than $T$ make a very small contribution to the response.

When $k_{z}=0$, the problem is similar to the one solved by Dawson and Fawcett [11], although in our problem, the Green's functions are obtained by superposing virtual acoustic sources placed in such a way as to generate the free or rigid surfaces of the fluid channel, and no restriction of the waveguide surface deformation is required when the deformation is inward. In addition, the solution is evaluated in the time domain by Fourier transforms, as well as being calculated in the frequency domain.

The BEM formulation is then described and the required Green's function presented, followed by a discussion of the numerical evaluation of the time signals. Numerical examples are given to illustrate the distribution of a pressure field generated by a dilatational point load inside a fluid channel in the presence of an irregular floor.

Obstacles of various sizes are analysed. Spectral representation of the signals is used to assess their features, which can be used as the basis for developing non-destructive testing and imaging methods.

\section{Problem statement}

Consider a cylindrical irregular inclusion of infinite extent, submerged in a spatially uniform fluid medium (Fig. 1), subjected to a harmonic point pressure load at position $\left(x_{0}, 0,0\right)$, oscillating with a frequency $\omega$.

$p_{\text {inc }}=\frac{A \mathrm{e}^{\mathrm{i}(\omega / \alpha)\left(\alpha t-\sqrt{\left(x-x_{0}\right)^{2}+y^{2}+z^{2}}\right)}}{\sqrt{\left(x-x_{0}\right)^{2}+y^{2}+z^{2}}}$

in which the subscript inc denotes the incident field, $A$ is the wave amplitude, $\alpha$ is the pressure wave velocity of the medium, and $i=\sqrt{-1}$.

Defining the effective wave numbers

$k_{\alpha}=\sqrt{\frac{\omega^{2}}{\alpha^{2}}-k_{z}^{2}}, \quad \operatorname{Im} k_{\alpha}<0$

by means of the axial wave number $k_{z}$, and Fourier-transforming Eq. (1) in the $z$-direction, one obtains

$\hat{p}_{\text {inc }}\left(\omega, x, y, k_{z}\right)=\frac{-\mathrm{i} A}{2} H_{0}^{(2)}\left(k_{\alpha} \sqrt{\left(x-x_{0}\right)^{2}+y^{2}}\right)$

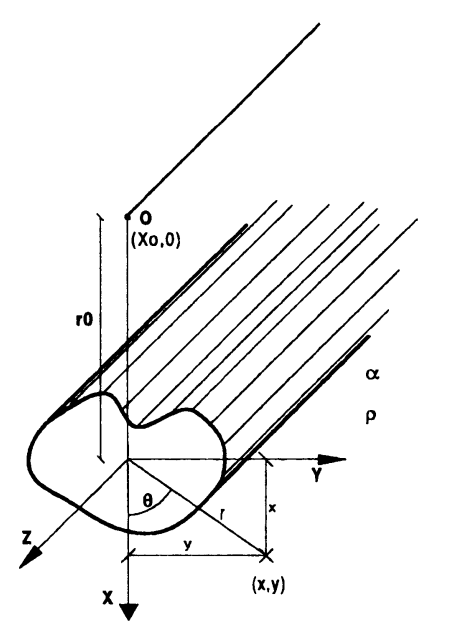

Fig. 1. Geometry of the problem. 
in which the $H_{n}^{(2)}(\cdots)$ are second Hankel functions of order $n$. By applying an Inverse Fourier Transform the former 3D pressure field can be obtained.

$p_{\text {inc }}(\omega, x, y, z)=\int_{-\infty}^{\infty} \hat{p}_{\text {inc }}\left(\omega, x, y, k_{z}\right) \mathrm{e}^{-\mathrm{i} k_{z} z} \mathrm{~d} k_{z}$

This continuous integral can be discretized by assuming the existence of an infinite number of sources placed along the $z$ direction at equal intervals, $L$. Thus, the incident field can be written as

$p_{\text {inc }}(\omega, x, y, z)=\frac{2 \pi}{L} \sum_{m=-\infty}^{\infty} \hat{p}_{\text {inc }}\left(\omega, x, y, k_{z}\right) \mathrm{e}^{-\mathrm{i} k_{z} z}$

with $k_{z}=(2 \pi / L) m$. This equation converges and can be approximated by a finite sum of terms. The distance $L$ needs to be large enough to avoid spatial contamination. In addition, the analyses use complex frequencies that further reduce the influence of the neighbouring fictitious sources.

Using this technique, the incident field generated by a point pressure load can be obtained as a discrete summation of 2D line loads with different values of $k_{z}$. In the same way, the scattered field originated by a point pressure load can be evaluated by solving a sequence of $2 \mathrm{D}$ problems. The same technique can be further extended to other load conditions, such as combined point loads.

The problem to be solved considers a spatially uniform inviscid fluid medium bounded by two flat surfaces, having a confined irregular deformation in its floor (inclusion). The pressure field defined by Eq. (3) needs to be reformulated to satisfy the boundary conditions at the surface and floor. The normal velocity must be null at the flat floor of the channel and at the rigid surface, under ice formations, in cold regions. On the other hand, the pressure reaches null values when the surface is open. This function can be achieved by superposing the pressure field generated by virtual sources with positive or negative polarity, and located so that the desired boundary conditions are ensured [22]. In the case of a free surface, the pressure field (Green's function $G\left(x, x_{0}, \omega\right)$ ) is given by the following expression:

$$
\begin{aligned}
G\left(x, x_{0}, \omega\right)= & \frac{-\mathrm{i}}{4}\left[H_{0}\left(k_{\alpha} r\right)\right]+\frac{-\mathrm{i}}{4}\left\{\sum _ { n = 0 } ^ { \mathrm { NS } } ( - 1 ) ^ { n } \left[H_{0}\left(k_{\alpha} r_{1}\right)\right.\right. \\
& \left.\left.-H_{0}\left(k_{\alpha} r_{2}\right)-H_{0}\left(k_{\alpha} r_{3}\right)-H_{0}\left(k_{\alpha} r_{4}\right)\right]\right\}
\end{aligned}
$$

while when the surface is rigid it takes the form

$$
\begin{aligned}
& G\left(x, x_{0}, \omega\right)= \\
& \quad \frac{-\mathrm{i}}{4}\left[H_{0}\left(k_{\alpha} r\right)\right]+\frac{-\mathrm{i}}{4}\left\{\sum _ { n = 0 } ^ { \mathrm { NS } } \left[H_{0}\left(k_{\alpha} r_{1}\right)+H_{0}\left(k_{\alpha} r_{2}\right)\right.\right. \\
& \left.\left.\quad+H_{0}\left(k_{\alpha} r_{3}\right)+H_{0}\left(k_{\alpha} r_{4}\right)\right]\right\}
\end{aligned}
$$

in which

$r=\sqrt{\left(x-x_{0}\right)^{2}+\left(y-y_{0}\right)^{2}}$

$r_{1}=\sqrt{\left(x-x_{0}\right)^{2}+\left(y+y_{0}+2 h n\right)^{2}}$

$r_{2}=\sqrt{\left(x-x_{0}\right)^{2}+\left(y-2 h-y_{0}-2 h n\right)^{2}}$

$r_{3}=\sqrt{\left(x-x_{0}\right)^{2}+\left(y+2 h-y_{0}+2 h n\right)^{2}}$

$h$ is the thickness of the channel.

The number of sources used (NS) is determined so that all the signals needed to define the signal within the time interval fixed by the frequency increment are taken into account.

\section{Boundary element formulation}

The BEM is used to obtain the 3D field generated by the scattering from a cylindrical inclusion with an irregular shape. In the case of an acoustic medium, the 2-1/2-D problem can be solved as a discrete summation of $2 \mathrm{D}$ BEM solutions for different $k_{z}$ wave numbers. Then, using the inverse Fourier transform, the 3D field can be synthesized. The wave number transform in discrete form is obtained, as explained above, by considering an infinite number of virtual point sources equally spaced along the $z$-axis and at a sufficient distance from each other to avoid spatial contamination [20].

Since the literature on the BEM is comprehensive, we do not give full details of the formulation required for the type of scattering problem presented here (see for example Ref. [23]). Next, we present a brief description of the BEM solution required to solve each $2 \mathrm{D}$ problem.

For frequency domain analysis, the acoustic pressure $(p)$ at any point of the spatial domain can be calculated making use of a single scalar equation, known as the Helmoltz equation

$\nabla^{2} p(\underline{x}, \omega)+k_{\alpha}^{2} p(\underline{x}, \omega)=0$

where $k_{\alpha}=\sqrt{\left(\omega^{2} / \alpha^{2}\right)-k_{z}^{2}}$. Considering a homogeneous fluid medium of infinite extent, containing an inclusion of volume $V$, bounded by a surface $S$, the boundary integral equations can be constructed by applying the reciprocity theorem, leading to

$$
\begin{aligned}
c p\left(\underline{x}_{0}, k_{z}, \omega\right)= & \int_{S} q\left(\underline{x}, \nu_{n}, \omega\right) G\left(\underline{x}, \underline{x}_{0}, k_{z}, \omega\right) \mathrm{d} s \\
& -\int_{S} H\left(\underline{x}, \nu_{n}, \underline{x}_{0}, k_{z}, \omega\right) p\left(\underline{x}, k_{z}, \omega\right) \mathrm{d} s
\end{aligned}
$$


In this equation $G$ and $H$ are, respectively, the fundamental solutions for the pressure $(p)$ and pressure velocity $(q)$, at $\underline{x}$ due to a virtual point load at $\underline{x}_{0}$. The factor $c$ is a constant defined by the shape of the boundary, receiving the value $1 / 2$ if $\underline{x}_{0} \in S$ and is smooth.

The boundary conditions prescribe null normal pressure velocities along the boundary $S$. Thus, Eq. (9) is simplified to

$c p\left(\underline{x}_{0}, k_{z}, \omega\right)=-\int_{S} H\left(\underline{x}, \nu_{n}, \underline{x}_{0}, k_{z}, \omega\right) p\left(\underline{x}, k_{z}, \omega\right) \mathrm{d} s$

Assuming the existence of an incident pressure wavefield striking the boundary, defined by Eq. (6) or Eq. (7), the following equation is derived

$$
\begin{aligned}
c p\left(\underline{x}_{0}, k_{z}, \omega\right)= & -\int_{S} H\left(\underline{x}, \nu_{n}, \underline{x}_{0}, k_{z}, \omega\right) p\left(\underline{x}, k_{z}, \omega\right) \mathrm{d} s \\
& +\hat{p}^{\text {inc }}\left(\underline{x}_{0}, k_{z}, \omega\right)
\end{aligned}
$$

The solution of this integral for an arbitrary boundary surface $(S)$ requires the discretization of the boundary into $N$ straight boundary elements, for which boundary values $p^{k}$ are ascribed. For constant value pressure boundary elements, Eq. (11) takes the form

$c p^{k}=-\sum_{l=1}^{N} H^{k l} p^{l}+p_{\mathrm{inc}}^{k}$

with $H^{k l}=\int_{C_{l}} H\left(\underline{x}_{l}, n_{l}, \underline{x}_{k}, k_{z}, \omega\right) \mathrm{d} s$, where $k$ is the loaded element, $p^{l}$ is the pressure in element $l$ and $H^{k l}$ is the pressure velocity component at $x_{l}$ due to a pressure load at $x_{k}$ and $n_{l}$ is the outward normal for the $l$ th boundary segment $C_{l}$. The required pressure velocity function $(H)$ is obtained by differentiating Eqs. (6) and (7) in relation to the unit outward normal.

The application of virtual loads along all boundary nodal points [Eq. (12), $k=1, N$ ] allows the definition of a linear system of equations that can be solved for the $N$ nodal pressures.

If the pressure is allowed to vary linearly within the boundary elements, using linear interpolation functions, then the number of nodes to be considered inside each element equals two. Twice the number of equations defined above are required, and the resulting integration then takes the form

$H^{k l}=\int_{C_{l}} \phi H\left(\underline{x}_{l}, n_{l}, \underline{x}_{k}, k_{z}, \omega\right) \mathrm{d} s$

where $\phi$ represents the required interpolation functions. Fig. 2 illustrates the interpolation functions and the position of the nodal points. Integrations in Eq. (13) are performed by means of Gauss-Legendre quadrature, using four integration points.

The scattered pressure field in the fluid is then defined as a

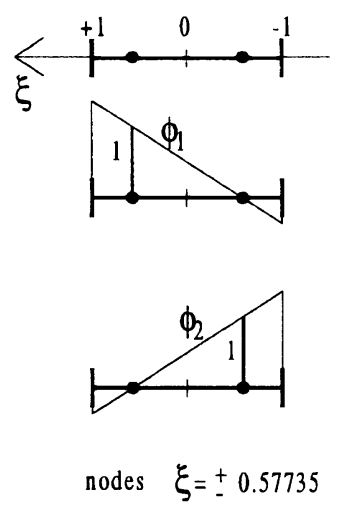

Fig. 2. Discontinuous linear boundary elements. Interpolating functions and nodal points position.

function of the nodal pressure values, as follows:

$p_{\mathrm{sca}}^{k}=\sum_{l=1}^{2 N} p^{l} H^{k l}$

where $p_{\mathrm{sca}}^{k}$ is the scattered pressure field at receiver $k, N$ the total number of boundary elements, and $p^{l}$ is the nodal pressure value at element $l$.

\section{Pressure in time-space}

The pressures in the time domain can be obtained by fast Fourier transform in $\omega$, taking the source whose temporal variation is given by a Ricker pulse. This pulse is chosen for its rapid decay, in both the time and frequency domains, allowing a reduction in the computational effort and easier interpretation of the results obtained in the time domain.

The Ricker function is given by the expression

$u(\tau)=A\left(1-2 \tau^{2}\right) \mathrm{e}^{-\tau^{2}}$

in which $A$ is the amplitude, $\tau=\left(t-t_{\mathrm{s}}\right) / t_{0}$ and $t$ represents the time, with $t_{\mathrm{s}}$ being the time when the maximum occurs, while $\pi t_{0}$ is the characteristic (dominant) wavelet period. Its

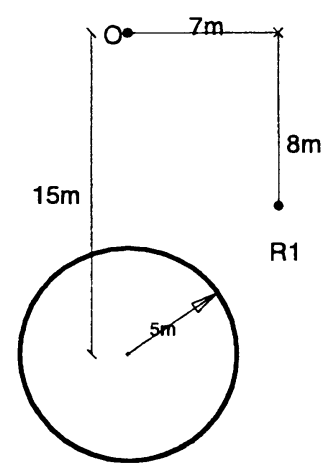

Fig. 3. Cylindrical inclusion in an unbounded medium. 


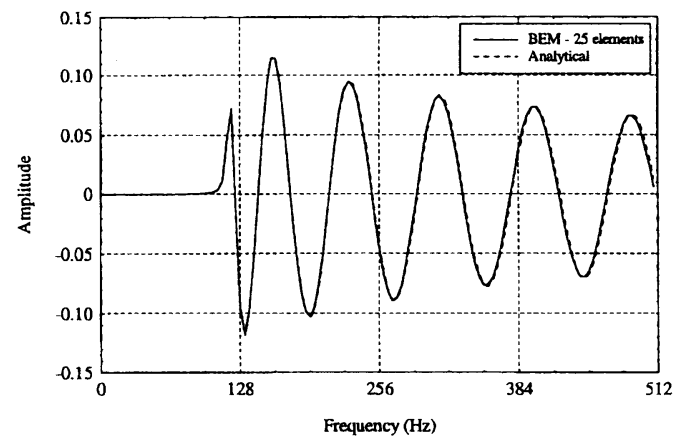

a)

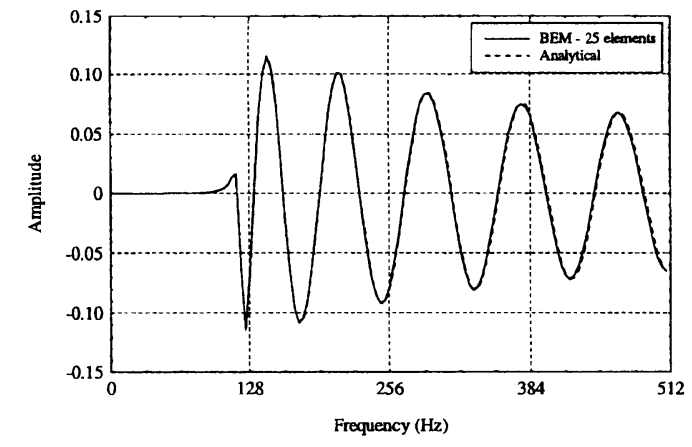

b)

Fig. 4. Fourier spectra: (a) real part; (b) imaginary part.

Fourier transform is

$$
U(\omega)=A\left[2 \sqrt{\pi t_{0}} e^{-i \omega t_{\mathrm{s}}}\right] \Omega^{2} \mathrm{e}^{-\Omega^{2}}
$$

where $\Omega=\omega t_{0} / 2$.

As stated before, the Fourier transformations are achieved by discrete summations over wave numbers and frequencies, which is mathematically equivalent to adding periodic sources at spatial intervals $L=2 \pi / \Delta k_{z}$ (in the $z$-axis), and temporal intervals $T=2 \pi / \Delta \omega$, with $\Delta k_{z}$ and $\Delta \omega$ being the wave number and frequency steps, respectively. The spatial separation $L$ must be large enough to prevent contamination of the response by the periodic sources. In other words, the contribution to the response by the fictitious sources must be guaranteed to occur at times later than $T$. Shifting the frequency axis slightly downward greatly helps achievement of this goal, that is, by considering complex frequencies with a small imaginary part of the form $\omega_{c}=\omega-\mathrm{i} \eta$ (with $\eta=0.7 \Delta \omega$ ). This technique results in a significant attenuation or virtual elimination of the periodic sources. In the time domain, this shift is later taken into account by applying an exponential window $e^{\eta t}$ to the response [24].

\section{Validation of the BEM algorithm}

The BEM algorithm was implemented and validated by applying it to a fixed cylindrical circular cavity, submerged in an unbounded homogeneous fluid medium $(\alpha=$ $1500 \mathrm{~m} / \mathrm{s}$ and $\rho=1000 \mathrm{Kg} / \mathrm{m}^{3}$ ), subjected to a harmonic point pressure load applied at point $O$, as in Fig. 3, for which the solution is known in closed form. Notice that Eqs. (6) and (7) were specialized to solve the present BEM problem by setting the NS parameter to zero.

The response is calculated for a single receiver placed at $x=7.0 \mathrm{~m}$ and $y=8.0 \mathrm{~m}$, for frequencies ranging from 4 to $512 \mathrm{~Hz}$, taking a constant value for $k_{z}=0.5 \mathrm{rad} / \mathrm{m}$. Twentyfive discontinuous linear boundary elements were used to

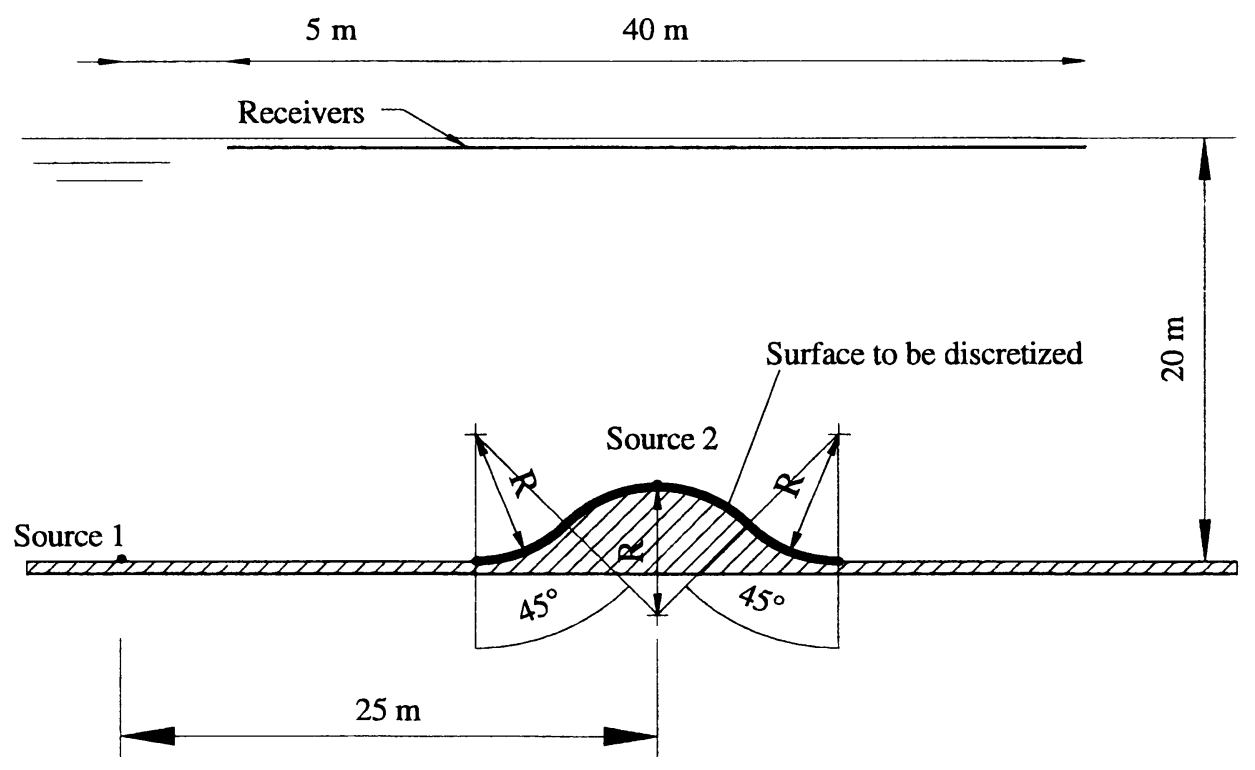

Fig. 5. Geometry of the channel and location of sources and receivers. 
model the inclusion. Fig. 4 displays the real and imaginary parts of the response for the numerical and analytical solutions, revealing a very good accuracy for the BEM approach. The comparison shows an excellent agreement for low frequencies, and only slight differences at high frequencies. This behaviour was expected, because the accuracy of the BEM solution depends on the ratio between the wavelength of the incident waves and the length of the boundary elements; in our example, this ratio decreases from $298(4 \mathrm{~Hz})$ to 3 $(512 \mathrm{~Hz})$ in the range of frequencies considered.

\section{Numerical examples}

All the cases presented refer to a channel, $20.0 \mathrm{~m}$ deep, with a confined irregular deformation located in its floor, as illustrated in Fig. 5. At the time $t=0$, the channel is struck from the rigid surface by a pressure source, placed directly above the deformation (source 2), or at a distance of $25.0 \mathrm{~m}$ horizontally, on the flat floor (source 1). Each source creates a spherical pressure pulse that propagates away from its origin. The pressure wave propagation velocity of $1500 \mathrm{~m} / \mathrm{s}$, and a host fluid density of $\rho=1000 \mathrm{~kg} / \mathrm{m}^{3}$ are constant for all the analyses. Computations are performed for variously sized confined deformations, defined with circular arcs of constant radius $(r=0.0,3.0,6.0$ and $9.0 \mathrm{~m})$. The pressure field generated by each source is determined along a line of receivers placed $0.2 \mathrm{~m}$ from the surface, and located on the same $x-y$ plane as the source.

The calculations are performed in the $8-512 \mathrm{~Hz}$ frequency range with $8 \mathrm{~Hz}$ increments. The source line dependence is a Ricker wavelet with a characteristic frequency of $150 \mathrm{~Hz}$. The frequency increment permits the dynamic analysis of the event for $0.125 \mathrm{~s}$, a value that is insufficient for the total development of the response. To permit analysis of the signal in time, a complex part was introduced into each excitation frequency to ensure damping in the response at the end of the time window considered $(1 / \Delta f=0.125 \mathrm{~s})$, as described previously. The distance between virtual sources, $L$, has been set to $2 \alpha(1 / \Delta f)=$ $375.0 \mathrm{~m}$.

The irregular part of the channel floor is modelled with a number of boundary elements that varies as a function of frequency, thereby maintaining a relation between the
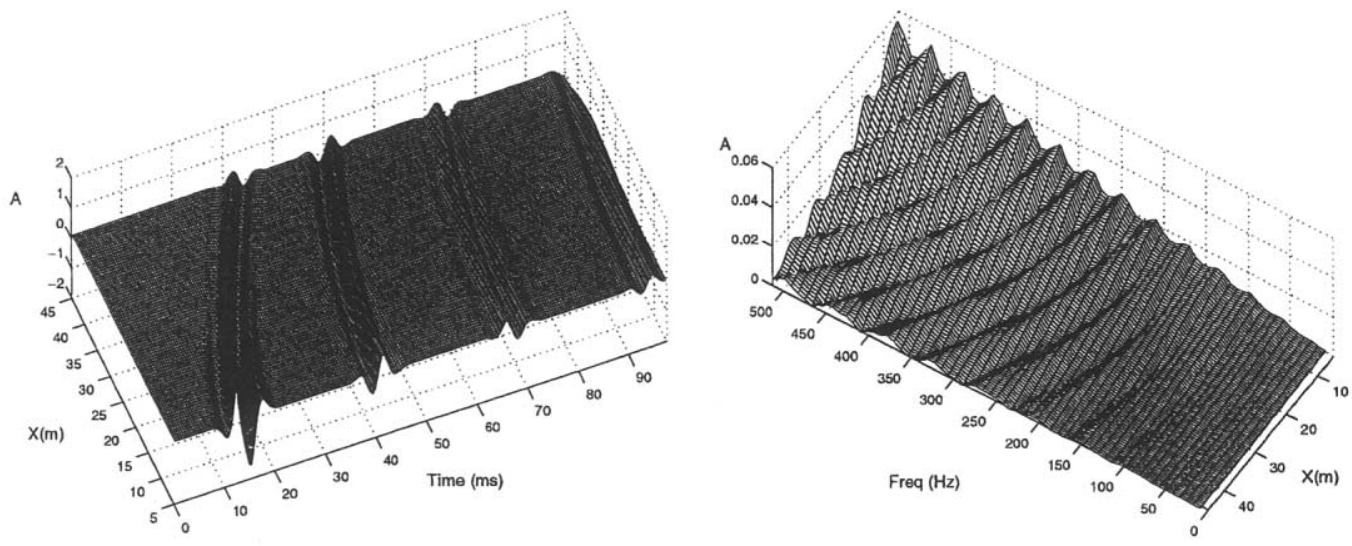

a)

b)
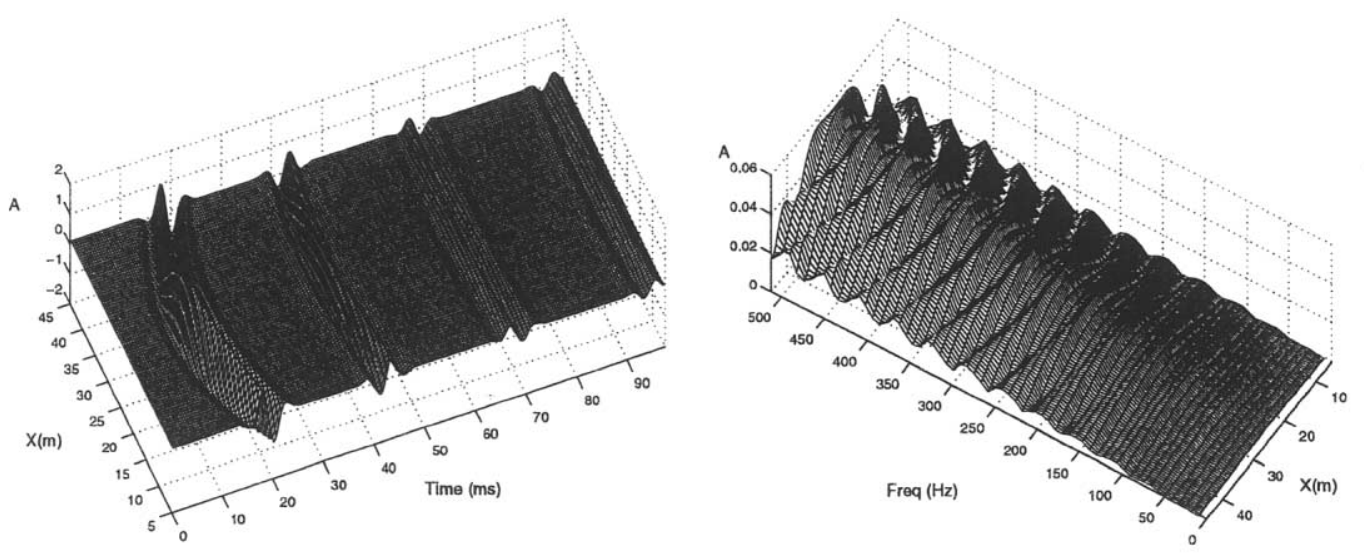

c)

d)

Fig. 6. Total response along the line of receivers when $R=0 \mathrm{~m}$ : (a) time response for source 1; (b) Fourier amplitude spectra for source 1; (c) time response for source 2; (d) Fourier amplitude spectra for source 2. 
incident wavelength and the length of the discretized element equal to a minimum of 6 . In no case is the number of nodal points less than 24. The surface and the flat part of the channel floor are not discretized, since the Green's function takes the boundary conditions at these interfaces into account.

Fig. 6a and c displays, for each source, the response recorded at the line of receivers, in the time domain, inside a channel with a perfectly flat floor $(R=0.0 \mathrm{~m})$. The first wave arrivals observed in these figures correspond to wave trains that are directly incident. These are followed by pulses of progressively lower amplitude, which are the result of reflections between the surface and the floor of the channel. Each time a pulse hits the surface it changes its phase $\left(180^{\circ}\right)$, while it conserves the phase when it impinges on the rigid floor. As these pulses reflect back and forth between the boundaries of the channel, they scatter and lose energy to the surrounding medium, and dissipate. One may also observe that the amplitude of the first arrivals clearly decreases when the distance between the receivers and the emission source increases. This is because the incident field takes more time to reach distant receivers, allowing the wavefront of its field to increase, and thus the energy to be dissipated.

Fig. $6 \mathrm{~b}$ and d displays the Fourier amplitude spectra $(0-$ $512 \mathrm{~Hz}$ ) of these responses. It may be noted that this plot exhibits pronounced peaks, which occur at well-defined frequencies. The periodicity of these peaks is caused by interference among reflections. This way, the distance in frequency between successive peaks is equal to $\Delta f=$ $\alpha / \Delta s$, in which $\Delta s$ is the different path among successive reflections $(\Delta s \approx 2 h)$. In the current example $\Delta f \approx 37.5 \mathrm{~Hz}$, as expected. The position of the peaks is related to the type of boundary. Since the base of the channel does not admit normal propagating velocities other than 0 , the first peak occurs for $f_{0}=\alpha / 4 h=18.75 \mathrm{~Hz}$.

Fig. 7 gives the response when sources 1 and 2 are excited in the presence of an irregular floor characterized by $R=$ $3.0 \mathrm{~m}$. When source 1 is excited, the time responses show that the first receivers continue to exhibit a response with a reasonable amplitude (Fig. 7a). However, additional pulses coming from the reflections occurring at the new boundary (the irregular floor) are observed. The signals recorded by the receivers furthest away suffer from the so-called
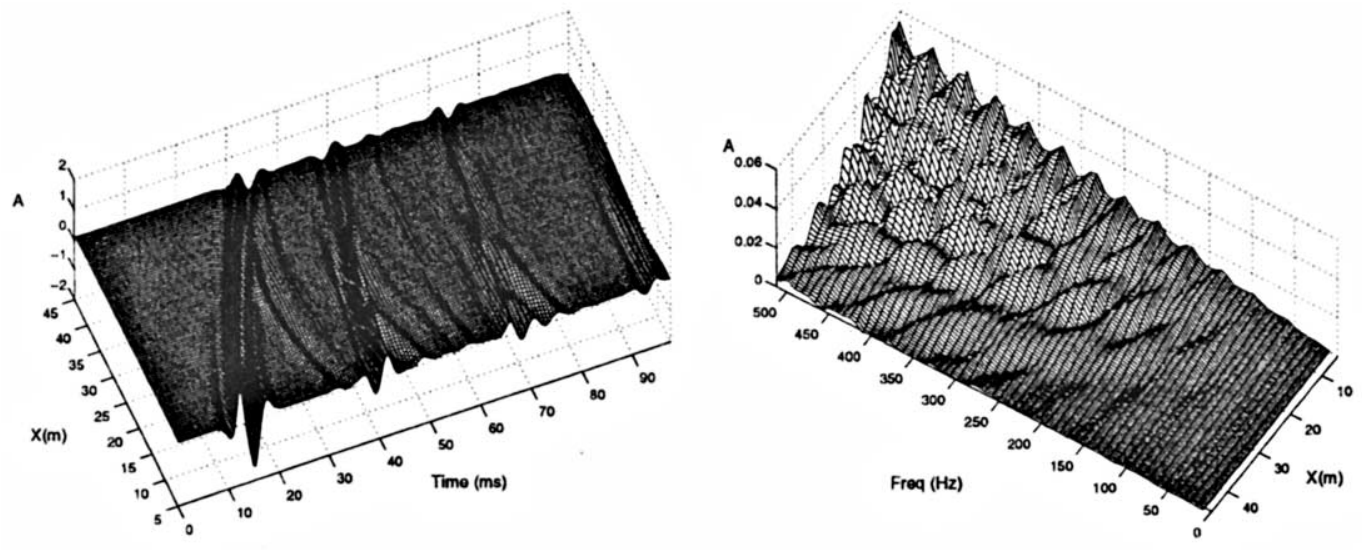

a)
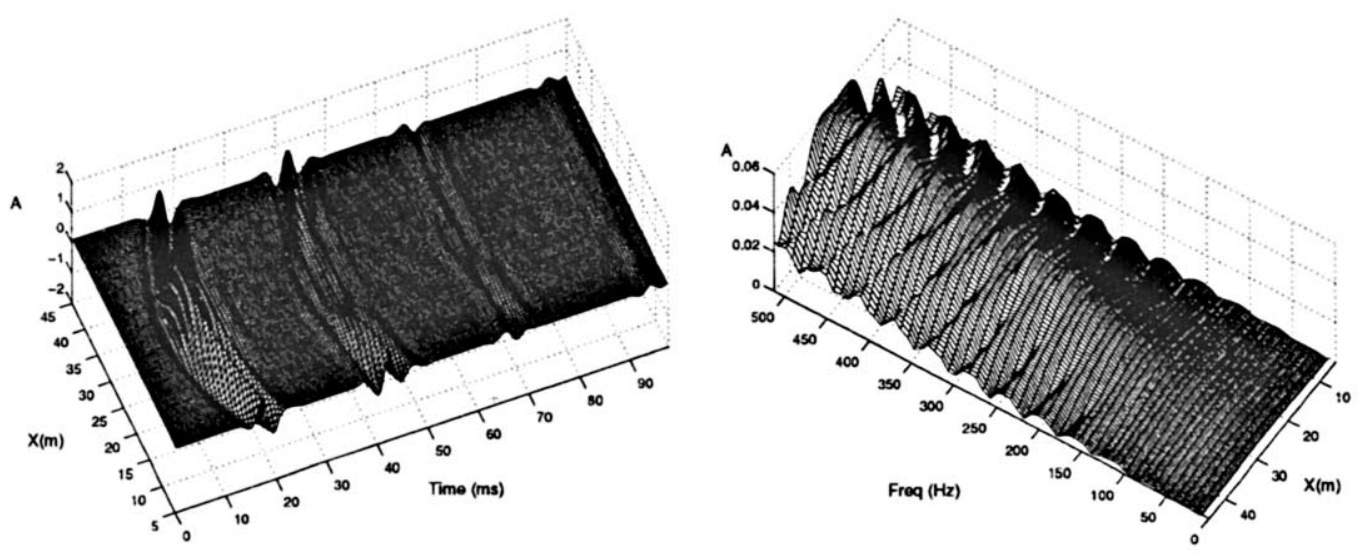

c)

Fig. 7. Total response along the line of receivers when $R=3 \mathrm{~m}:$ (a) time response for source 1; (b) Fourier amplitude spectra for source 1; (c) time response for source 2; (d) Fourier amplitude spectra for source 2. 
'shadow effect', caused by the obstacle. As a result, the amplitudes of the second order pulses show a slight decrease in relation to the signals given in Fig. 6. The signals generated when the excited source is at position 2 are symmetrical in relation to this position, as was expected (Fig. 7c). The receivers placed in the vicinity of the floor deformation show smaller amplitudes than do the receivers that are further away. This phenomenon is explained in the light of the reflective power of the convex shape of the obstacle, which enables energy to be reflected to the sides, away from the central zone, where the source is located.

Fig. $7 \mathrm{~b}$ and $\mathrm{d}$ shows the Fourier amplitude spectra of the responses. Once again, these graphs at low frequencies show a sequence of peaks in well-defined positions, as a function of the height of the liquid surface. Additional peaks, resulting from the presence of the obstacle, can also be observed as the frequency increases owing to the interference between reflections. The Fourier spectra responses recorded above the deformation, when source 2 is excited, allow the depth of the obstacle to be evaluated $(18.24 \mathrm{~m})$. These peaks are not very pronounced because the shape of the obstacle allows the energy to spread to the sides. At higher frequencies, one can clearly see the presence of these peaks, which tend to be $\Delta f \approx 41.1 \mathrm{~Hz}$ apart $(\Delta f=\alpha / \Delta s \approx 1500 /(2 \times 18.24))$. A geometric acoustic ray analysis shows that these signals result from the propagation of a pulse that travels between the upper part of the deformation and the free surface. The travel times coincide with the arrival of the signals in the time domain ( $t=12.2 \mathrm{~ms}, t=36.5 \mathrm{~ms}$, and $t=60.8 \mathrm{~ms}$ ), illustrated in Fig. 7c.

The presence of the deformation is not noticeable at low frequencies. All receivers exhibit a Fourier spectrum with a peak positioned at $f_{0}=\alpha / 4 h=18.75 \mathrm{~Hz}$ due to reflections from the flat floor. This can be explained by the fact that, at low frequencies, the wavelength of the incident field is very large compared with the size of the deformation, so the waves do not detect the presence of the irregular floor.

Fig. 8 illustrates the responses obtained when the deformation reaches $R=6.0 \mathrm{~m}$ and $R=9.0 \mathrm{~m}$, and the source is placed at position 2 . These signals have features similar to those observed in the previous case. Again, at very low frequencies the presence of the irregular floor is not noticeable. However, as the size of the deformation increases, its
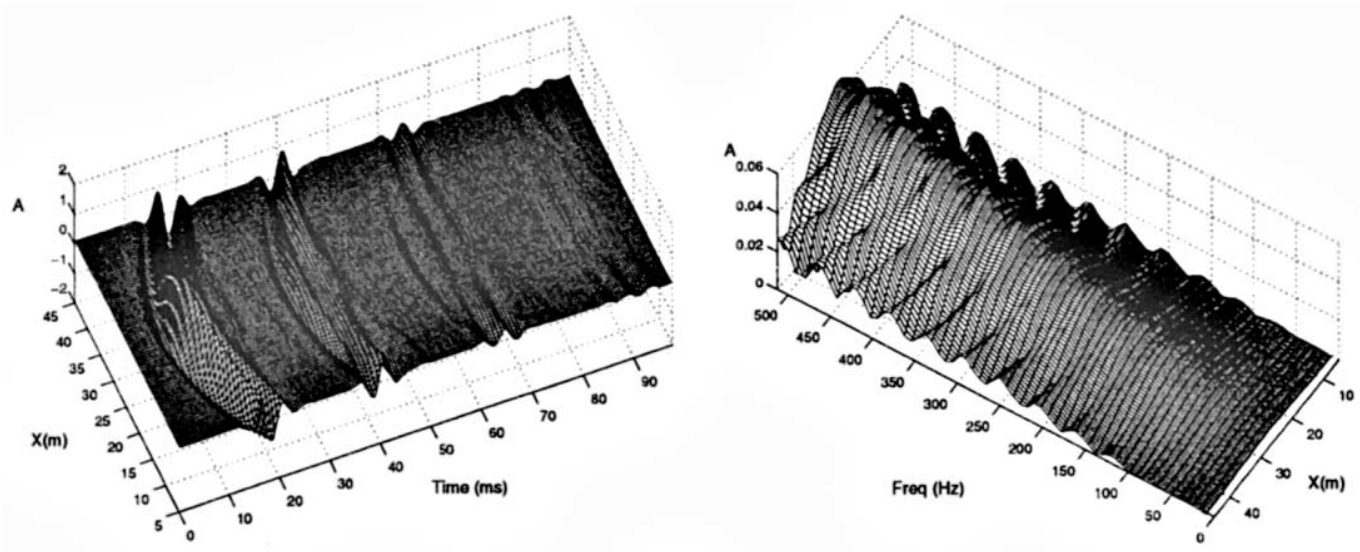

a)
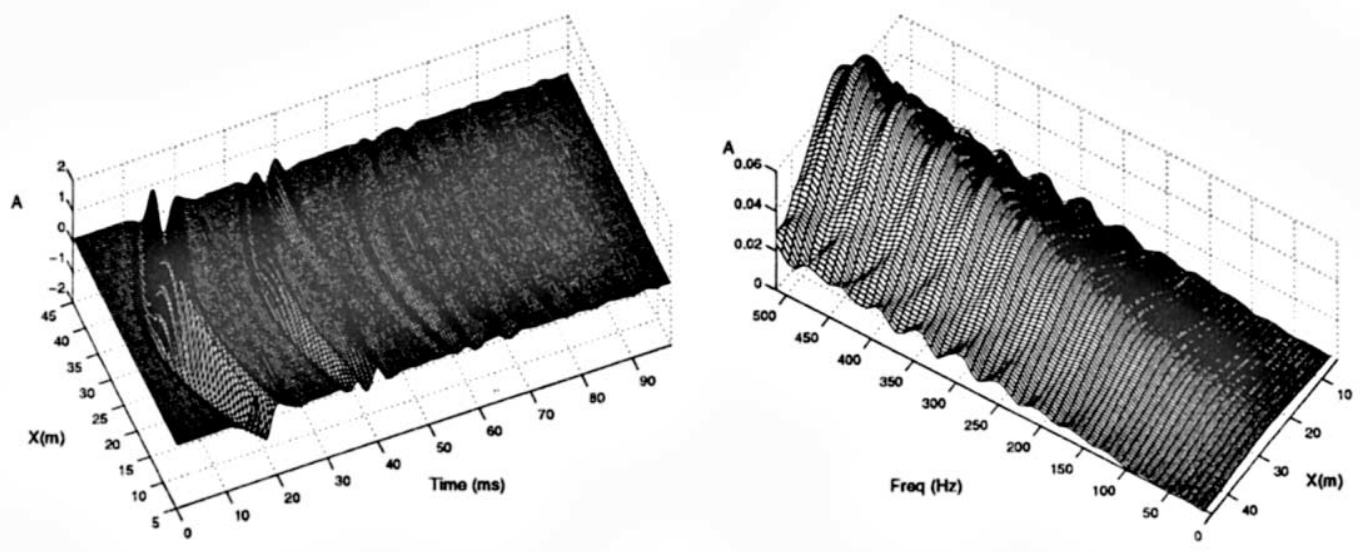

c)

d)

Fig. 8. Total responses when source 2 is excited: (a) time response for a deformation $R=6 \mathrm{~m}$; (b) Fourier amplitude spectra for a deformation $R=6 \mathrm{~m}$; (c) time response for a deformation $R=9 \mathrm{~m}$; (d) Fourier amplitude spectra for a deformation $R=9 \mathrm{~m}$. 


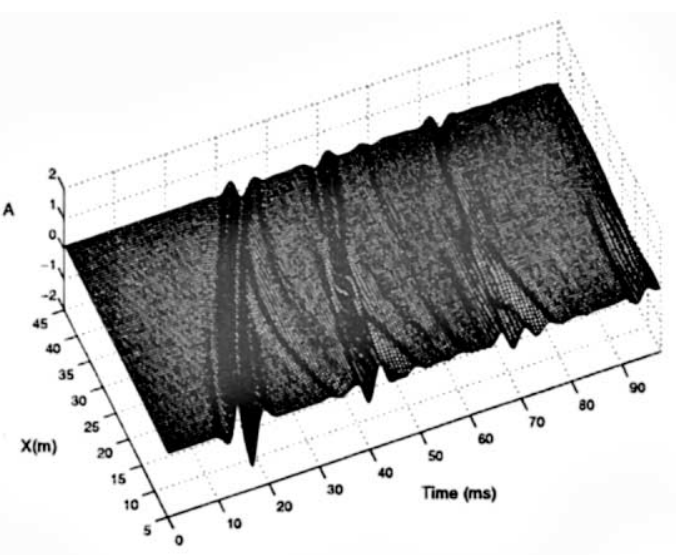

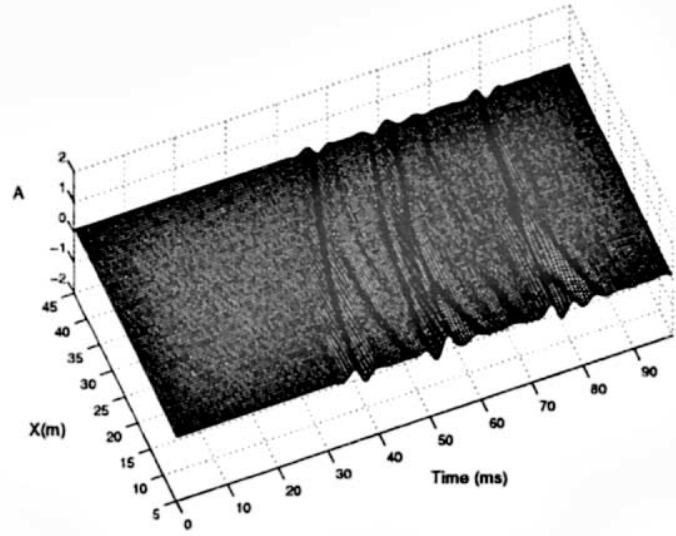

b)

Fig. 9. Time response for a deformation $R=3 \mathrm{~m}$. when source 1 is excited: (a) $Z=0 \mathrm{~m}$; (b) $Z=50 \mathrm{~m}$.

presence can be detected sooner in the frequency domain (see Figs. 7d, 8b and d). At higher frequencies, the receivers placed above the floor deformation exhibit a frequency spectrum with peaks that tend to be located 45.5 and $50.9 \mathrm{~Hz}$ apart, revealing the height of the water above the deformations (16.5 and $14.7 \mathrm{~m})$, when the irregular floor is characterized by $R=6.0 \mathrm{~m}$ and $R=9.0 \mathrm{~m}$, respectively. The time records exhibit a sequence of pulses that may appear complicated, but the arrival times of the various pulses at the receivers can be understood in terms of the ray theory of geometric acoustics. It is also observed that bigger deformations allow more energy to be reflected. This is shown by the amplitude of the second order reflection pulses.

Responses were also obtained for the receivers for $x-y$ planes $z=10.0 \mathrm{~m}$ and $z=50.0 \mathrm{~m}$ (as in Fig. 9) for a deformation with $R=3.0 \mathrm{~m}$. Again, time responses can be interpreted as a series of pulses, corresponding to reflections from the surface and bottom of the waveguide and from the obstacle. It is clear that the arrival of the first pulses at receivers placed at $z=50.0 \mathrm{~m}$ occurs much later and with smaller amplitudes than for receivers placed at $z=10.0 \mathrm{~m}$. As the distance in $z$ increases, receivers behind the obstacle seem less influenced by its presence.

When source 1 is excited, the 'shadow zone' increases in importance in the presence of bigger floor deformations. The creation of the shadow zones also depends on the excitation frequency. Higher frequencies are more affected by the existence of inclusions than lower frequencies. The phenomenon of shadow zone creation was approached by evaluating responses over a fine grid, defined as illustrated in Fig. 10. Responses were calculated for frequencies of 25, 125 and $250 \mathrm{~Hz}$. Fig. 11 shows the response over two vertical grids of receivers placed at $z=0.0 \mathrm{~m}$ and $z=50.0 \mathrm{~m}$, respectively. As expected, the results obtained for the lower frequency do not show any significant influence from the presence of the deformation. As the frequency increases, a shadow zone clearly shows up behind the obstacle, becoming more evident for the higher frequency $(250 \mathrm{~Hz})$. By comparing the response over the two grids, it is possible to conclude that this shadow effect is much less significant for $z=50.0 \mathrm{~m}$, as the amplitude of the response behind the obstacle becomes comparatively higher. This can be explained if we appreciate that the travel path followed by acoustic rays in order to hit receivers in this grid is less disturbed by the presence of the deformation than it is for those placed at $z=0.0 \mathrm{~m}$, thus allowing comparatively more energy to reach the zone behind the obstacle.

\section{Conclusions}

A discrete integration over wave numbers and frequencies has been used to compute the $3 \mathrm{D}$ scattered pressure from a compact deformation located on the floor of a fluid channel. The required integrations are restricted to the surface of the deformation, as a result of using the appropriate Green's functions, built by superposing virtual acoustic sources. The time responses were obtained by Fourier transformation using complex excitation frequencies. No restriction of the surface deformation is required when the deformation is inward.

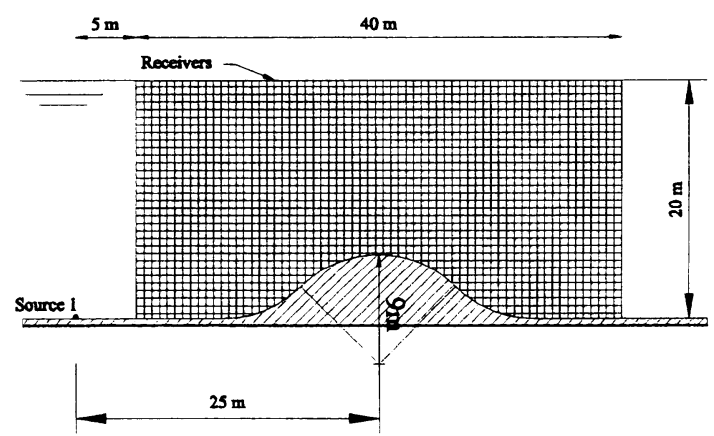

Fig. 10. Geometry of the problem $(R=9 \mathrm{~m})$. 

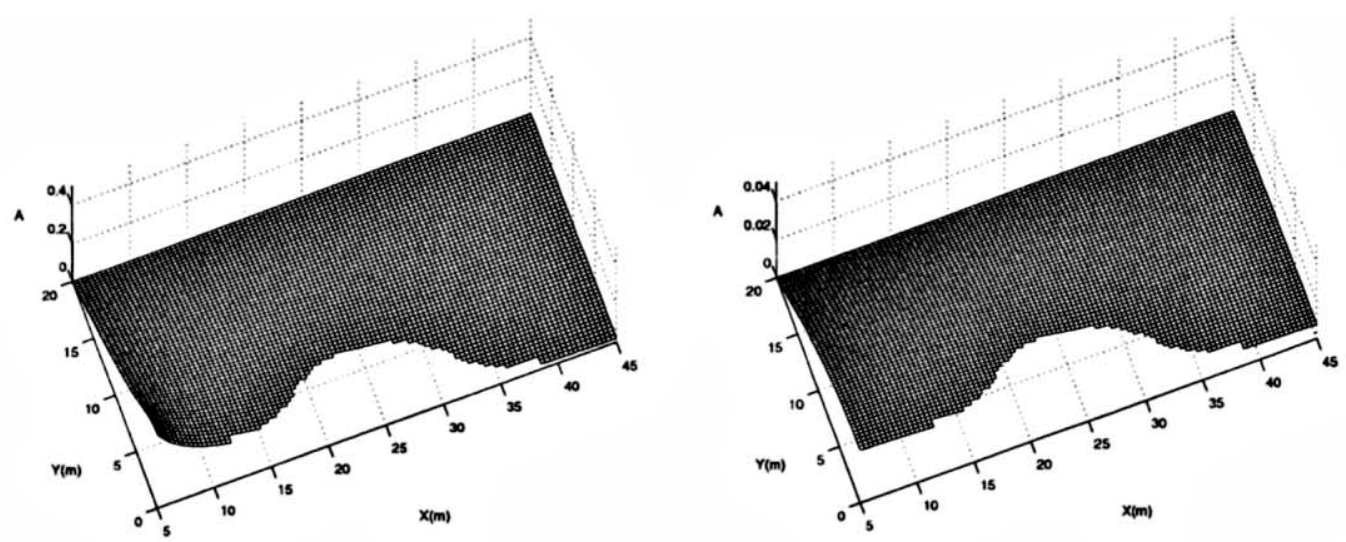

a)
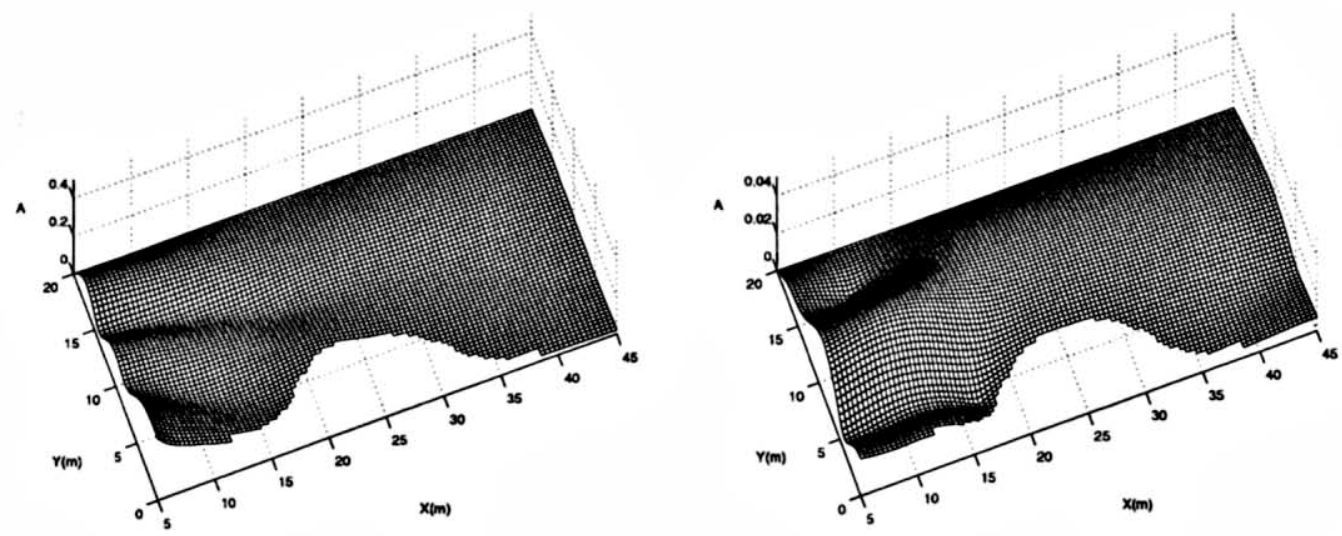

b)
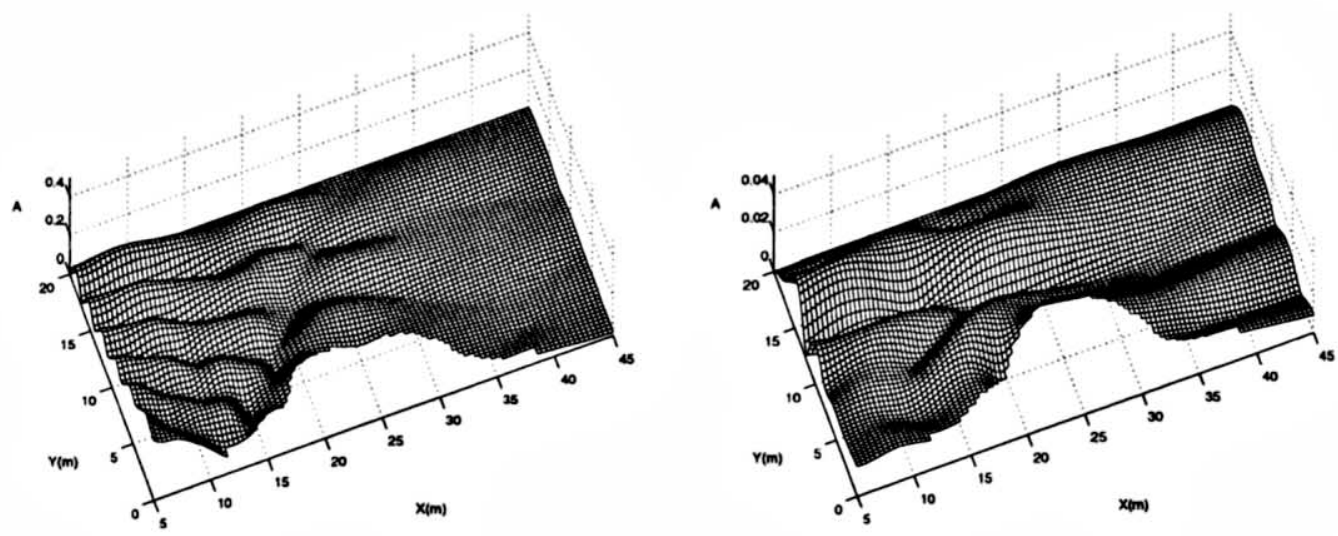

c)

Fig. 11. Steady state responses. $Z=0 \mathrm{mv} Z=50 \mathrm{~m}$ : (a) $25 \mathrm{~Hz}$; (b) $125 \mathrm{~Hz}$; (c) $250 \mathrm{~Hz}$.

The main features of the way the pressure field generated by a pressure load changes inside a fluid channel, in the presence of an irregular floor, are described. The pressure field inside a channel generated by dynamic excitation sources is markedly changed in the presence of obstacles placed on its base. These alterations permit the definition of shadow zones, which have little energy, in the zones of the channel opposite to where the excitation sources are located.
This effect becomes less evident as the distance in $z$ increases.

The examples described above made it possible to confirm the reflective power of the convex part of these obstacles. In addition, it was observed that the response spectrum for the excitation frequencies and size of the obstacles considered clearly showed the height of the surface of the liquid in the channel. 


\section{References}

[1] Özdogan Y. Seismic data processing. SEG Press, 1987.

[2] Claerbout JF. Fundamentals of geophysical data processing. New York: McGraw-Hill, 1976.

[3] Cordier J-P. Velocities in reflection Seismology. Holland: D. Reidel Publishing Company, 1985.

[4] Godkin Carl B, Travel time inversion of multi-offset vertical seismic profiles, Master thesis Massachusetts Institute of Technology, Cambridge, Massachusetts, 1985.

[5] Griffiths DH, King RF. Applied geophysics for geologists and engineers. Oxford: Pergamon Press, 1981. p. 1-69.

[6] Pao YH, Mow CC, Diffraction of elastic waves and dynamic stress concentrations. Crane and Russak, 1973.

[7] Stephens RA. A review of finite difference methods for seismoacoustic problems at the seafloor. Rev Geophys 1988;26:445-58.

[8] Marfurt KJ. Accuracy of finite-difference and finite-element modeling of the scalar and elastic wave equations. Geophysics 1984;49:53349.

[9] Kausel E, Forced vibrations of circular foundations in layered media, MIT Research Report 70-3, Department of Civil Engineering, Massachusetts Institute of Technology, Cambridge, MA, 1974

[10] Holford RL. Scattering of sound waves at a periodic pressure-release surface: An exact solution. J Acoust Soc Am 1981;70:1116-28.

[11] Dawson TW, Fawcett JA. A boundary integral equation method for acoustic scattering in a waveguide with nonplanar surfaces. J Acoust Soc Am 1990;87:1110-25.

[12] Ahluwalia DS, Keller JB. Exact and asymptotic representations of the sound field in a stratified ocean. In: Keller JB, Papadakis JS, editors. Wave propagation and underwater acoustics. New York: Springer, 1977. p. $14-85$.
[13] Fawcett JA. The computation of the scattered pressure field from a cylinder embedded between two half-spaces with different densities. J Acoust Soc Am 1996;99:2435-8.

[14] Fawcett JA. Acoustic scattering from cylindrical objects embedded between two half-spaces. J Acoust Soc Am 1996;100:3053-60.

[15] Dravinski M, Mossessian TK. Scattering of plane harmonic P, SV and Rayleigh waves by dipping layers of arbitrary shape. Bull Seism Soc Am 1987;77:212-35.

[16] Pedersen HA, Sanchez-Sesma FJ, Campillo M. Three-dimensional scattering by two-dimensional topographies. Bull Seism Soc Am 1994;8:1169-83.

[17] Sanchez-Sesma FJ. Diffraction of elastic waves by three-dimensional surface irregularities. Bull Seism Soc Am 1983;83:1621-36.

[18] Beskos DE. Wave propagation through ground. In: Manolis GD, Davies TG, editors. Boundary element techniques in Geomechanics. Southampton: Computational Mech Publ, 1993. p. 359-406 (Elsevier Applied Science, London).

[19] Tadeu A, Godinho L. 3D wave scattering by a fixed cylindrical inclusion submerged in a fluid medium. Engng An Bound El 1999;23:74556.

[20] Bouchon M, Keiti A. Discrete wave-number representation of seismic source wavefields. Bull Seism Am 1977;67:259-77.

[21] Phinney RA. Theoretical calculation of the spectrum of first arrivals in layered elastic mediums. J Geophys Res 1965;70:5107-23.

[22] Tadeu AJB, Modelling and seismic imaging of buried structures, $\mathrm{PhD}$ thesis, MIT, Department of Civil Engineering, Cambridge, Massachusetts, 1992

[23] Manolis GD, Beskos DE. Boundary element methods in Elastodynamics. London: Unwin Hyman (sold to Chapman and Hall), 1988.

[24] Kausel E, Roesset JM. Frequency domain analysis of undamped systems. J Engng Mech, ASCE 1992;118(4):721-34. 